\title{
New transient Galactic bulge intermediate polar candidate XMMU J175035.2-293557
}

\author{
F. Hofmann ${ }^{1}$, G. Ponti ${ }^{1}$, F. Haberl ${ }^{1}$, and M. Clavel ${ }^{2}$ \\ 1 Max-Planck-Institut für extraterrestrische Physik, Giessenbachstraße, 85748 Garching, Germany \\ e-mail: fhofmann@mpe.mpg.de \\ 2 Univ. Grenoble Alpes, CNRS, Institut de Planétologie et d'Astrophysique de Grenoble (IPAG), 38000 Grenoble, France
}

Received 26 February 2018 / Accepted 14 June 2018

\begin{abstract}
Context. For the past decades, a rare subclass of cataclysmic variables (CV), with magnetised white dwarfs (WD) as accretors, has been studied. They are called intermediate polars (IP) and have been suggested to be the main contributors to the diffuse, hard X-ray emission close to the Galactic center (GC) and in the Galactic bulge (GB), because most of them are unresolved there.

Aims. In an ongoing X-ray survey $\left(0.5-10 \mathrm{keV}\right.$ energy band) of $3^{\circ} \times 3^{\circ}$ around the GC with the XMM-Newton observatory, we conducted a systematic search for transient X-ray sources.

Methods. Promising systems were analyzed for spectral, timing, and multiwavelength properties to constrain their nature.

Results. We discovered a new highly variable (factor $\gtrsim 20$ ) X-ray source about $1.25^{\circ}$ south of the GC. We found evidence that makes the newly discovered system a candidate IP. The X-ray light curve shows a period of $511 \pm 10 \mathrm{~s}$, which can be interpreted as the spin period of the WD. The X-ray spectrum is well fit by a bremsstrahlung model with a temperature of $13.9 \pm 2.5 \mathrm{keV}$, suggesting a WD mass of $0.4-0.5 M_{\odot}$. We were unable to identify a blue optical counterpart in any of the candidate sources, as would be expected for IPs.

Conclusions. The high X-ray absorption and absence of a clear optical counterpart suggest that the source is most likely located in the GB. This would make the system a transient IP (GK Per class) with an especially high peak X-ray luminosity, which means that it is a very faint $\mathrm{X}$-ray transient.
\end{abstract}

Key words. X-rays: binaries - Galaxy: center - Galaxy: bulge - white dwarfs - novae, cataclysmic variables

\section{Introduction}

Intermediate polars (IP) are a subclass of magnetic cataclysmic variables (CV) where a white dwarf (WD) is accreting mass from a late-type donor star (see, e.g., Downes et al. 2001; Mukai 2017). The accretion disc around the WD is interrupted by the magnetic field and matter is accreted along the field lines onto the magnetic poles of the WD. X-ray emission is created in accretion curtains onto the magnetic poles and the accretion disc of the WD (e.g., Patterson 1994; Hellier 2007; Barbera et al. 2017). IPs usually show light-curve modulations (X-ray and optical) by the spin of the WD and the orbital period around its donor star (see, e.g., Watson et al. 1985; Osborne 1988; Cropper 1990; Kim \& Beuermann 1995). About 50 IPs have been confirmed, and more than 100 candidates are known today (see the IP catalogue maintained by Koji Mukai ${ }^{1}$ ).

Recently, IPs have been discussed as the origin of the hard ( 2-40 keV range) X-ray emission close to the Galactic center (GC) and in the Galactic bulge (GB) region (e.g., Krivonos et al. 2007; Revnivtsev et al. 2009; Perez et al. 2015; Hailey et al. 2016). Pretorius \& Mukai (2014) estimated the space density of IPs from a Swift-BAT survey (14-195 keV), where they are brighter than in the $0.5-10 \mathrm{keV}$ band because of their high temperature X-ray spectrum. Muno et al. (2004), Laycock et al.

\footnotetext{
1 https://asd.gsfc.nasa.gov/Koji.Mukai/iphome/
}

(2005), and Ruiter et al. (2006) derived from a deep Chandra survey that most of the fainter, hard GC X-ray sources should be IPs. Investigating the population of IPs in the GB/GC area is therefore very important for understanding their contribution to the diffuse X-ray emission (Hong 2012). In addition, IPs trace the CV population (see Cropper 1990), which is important for understanding the GB stellar composition (e.g., Calamida et al. 2014, 2015).

Because IPs are relatively faint in X-rays and optical and because of the high extinction toward the GC, deep and high spatial resolution observations are needed. We used the extension of the deep XMM-Newton GC X-ray survey (about 0.5-10 keV energy band, Ponti et al. 2015) to the GB (Ponti et al., in prep.) to search for transients and highly variable sources (overall $\sim 3^{\circ} \times 3^{\circ}$ ). We detected a new X-ray source $1.25^{\circ}$ south of Sgr $\mathrm{A}^{\star}$ (in Galactic coordinates). The analysis of the X-ray spectrum, search for periodic modulations, and lack optical counterpart identification make the source an IP candidate that is most likely located in the GB. This is observed very rarely (see Hong et al. 2009; Britt et al. 2013; Torres et al. 2014; Johnson et al. 2017). A larger sample of clearly identified IPs in the GB is required for direct population constraints. As a luminous, transient IP, the source would be the second member of the GK Per class (see the recent description by Yuasa et al. 2016), and it might have a subgiant donor star. 


\section{X-ray data}

The analysis in this work is based on a $25 \mathrm{ks}$ XMM-Newton observation (ObsID: 0801681401, start date 2017-10-07, PI: Ponti). We used data from the XMM-Newton European Photon Imaging Camera (EPIC) PN (Strüder et al. 2001), and MOS CCDs (Turner et al. 2001). The spectral fitting was performed using XSPEC (version 12.9.1n, Arnaud 1996) and the Bayesian fitting package BXA (Buchner et al. 2014). The data reduction was performed using the XMM-Newton Science Analysis System (SAS) version 16.1.0. Long-term source variability information was obtained together with previous $15 \mathrm{ks}$ and 2 ks Chandra (for a description, see Garmire et al. 2003) observations (ObsIDs: 7167, start date 2006-10-30, PI: Grindlay; 8753, start date 2008-05-13, PI: Jonker), which were part of the Chandra Galactic bulge survey (Jonker et al. 2011). These observations were reprocessed using the Chandra Interactive Analysis of Observations software package (CIAO; Fruscione et al. 2006) version 4.5 and the Chandra Calibration Database (CalDB; Graessle et al. 2007) version 4.5.9. Uncertainties are quoted on the $1 \sigma$ level unless stated otherwise.

\section{Results}

The source position is RA: $17^{\mathrm{h}} 50^{\mathrm{m}} 35.2^{\prime \prime}$ Dec: $-29^{\circ} 35^{\prime} 57.2^{\prime \prime}$ with an uncertainty of $1.2^{\prime \prime}$ (systematic, see XMM-Newton technical note: XMM-SOC-CAL-TN-0018) plus 0.5" (statistical), leading to $1.3^{\prime \prime}$ total $1 \sigma$ uncertainty. A cross correlation of detected sources in the XMM-Newton observation with the Chandra Galactic Bulge survey catalogue of X-ray and optical sources (Jonker et al. 2014; Wevers et al. 2016) showed an average offset of less than $1^{\prime \prime}$ in both RA and Dec direction.

\subsection{X-ray spectrum and variability}

We extracted the source and background spectra $(0.5-10 \mathrm{keV})$ from two circles of $25^{\prime \prime}$ radius shown in Fig. 1, using standard event file filtering and grouped the spectrum to contain at least 22 counts per bin. The background region was chosen to contain the same level of enhanced X-ray emission as the source region (caused by extended recombining plasma south of the GC, see Nakashima et al. 2013). The XMM-Newton EPIC spectrum contains $\sim 6 \times 10^{3}$ net counts $(0.5-10 \mathrm{keV}$ range). Table 1 shows the best-fit parameters and goodness of fit $\left(\chi^{2}\right)$ for several absorbed single-component spectral models. The fitted parameters were obtained using the BXA fitting software with wide and flat priors. For every tested parameter combination, the model flux was calculated. The lower limit, best-fit value, and upper limit are the 15,50 , and 85 percentiles of the parameter distributions (transformed into symmetric uncertainties). The analysis shows that bremsstrahlung (bremss) is the best-fit model compared to a disc-blackbody (diskbb, $\Delta \chi^{2}=37$ ) or power law (pow, $\Delta \chi^{2}=354$ ). A collisionally ionised plasma (apec) model with solar abundance would also fit the data well (see Table 1). There is no evidence for an Fe-line complex in the $6-7 \mathrm{keV}$ range. We obtained $3 \sigma$ upper limits on the equivalent width (EW) of emission lines at energies: $\mathrm{EW}(6.4 \mathrm{keV}) \lesssim 400 \mathrm{eV}$, $\mathrm{EW}(6.7 \mathrm{keV}) \lesssim 220 \mathrm{eV}$, and $\mathrm{EW}(7.0 \mathrm{keV}) \lesssim 450 \mathrm{eV}$ (main peaks of the Fe-line complex, see, e.g., Hellier \& Mukai 2004) by adding additional narrow Gaussian lines to the bremss model. The measured foreground column density of neutral hydrogen was fit as $\mathrm{N}_{\mathrm{H}} \approx(1.2 \pm 0.1) \times 10^{22} \mathrm{~cm}^{-2}$ using the TBabs model with cross-sections and abundances from Wilms et al. (2000).

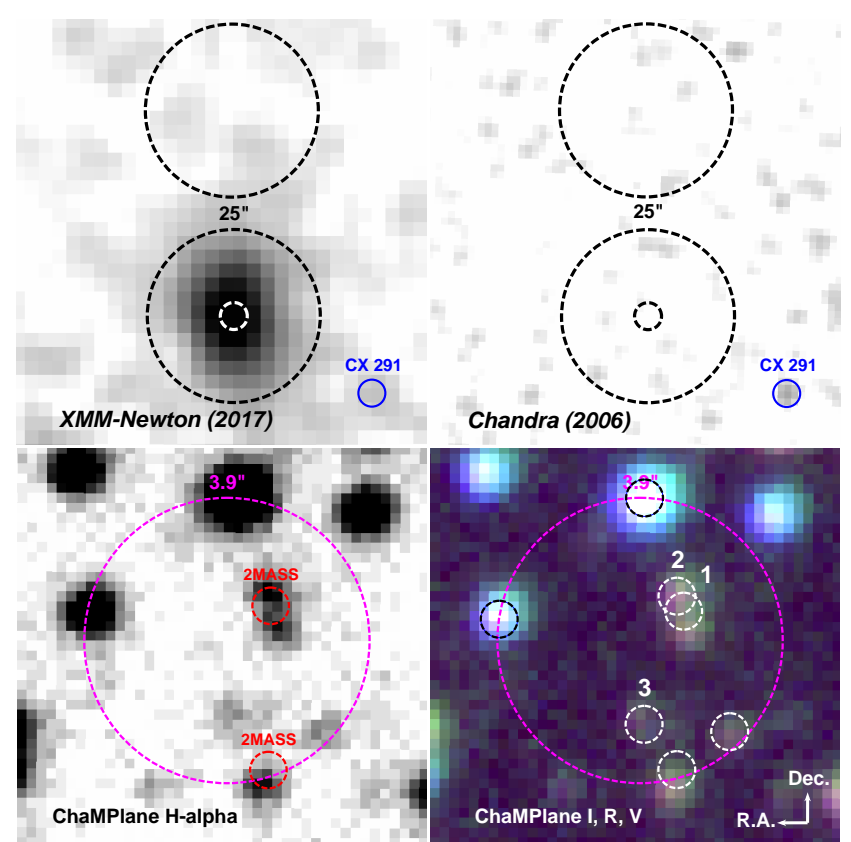

Fig. 1. Top left: XMM-Newton EPIC 1-2 keV flux image (from 2017$10-07,4 \times 4$ " pixel size, smoothed by a 2-pixel Gaussian). Circular extraction regions (25" radius) of source and background counts (black dashed), $3 \sigma$ positional uncertainty (white dashed). Top right: Chandra ACIS-I 1-2 keV flux image (from 2006-10-30, $2 \times 2^{\prime \prime}$ pixel size, smoothed by a 2-pixel Gaussian). The blue circle indicates a source from the Jonker et al. (2014) Chandra catalogue. Bottom left: zoomed ChaMPlane (Grindlay et al. 2003) $\mathrm{H}_{\alpha}$ image (red circles: 2MASS sources, magenta circle: $3 \sigma \mathrm{X}$-ray positional uncertainty). Bottom right: ChaMPlane colour band image (red: $I$, green: $R$, blue: $V$ ). Small circles are correlated sources from Wevers et al. (2016), and the three closest are numbered.

Table 1. XMM-Newton spectral model parameters $(0.5-10 \mathrm{keV}$ range).

\begin{tabular}{lllll}
\hline \hline & pow $^{a}$ & diskbb $^{a}$ & bremss $^{a}$ & apec $^{a, c}$ \\
\hline$N_{\mathrm{H}}{ }^{b}$ & $3.6 \pm 0.2$ & $1.0 \pm 0.1$ & $1.2 \pm 0.1$ & $1.3 \pm 0.1$ \\
$k T^{b}$ & - & $2.2 \pm 0.1$ & $13.9 \pm 2.5$ & $15.5 \pm 2.5$ \\
$\Gamma^{b}$ & 2.8 & - & - & - \\
$F_{\mathrm{X}}{ }^{b}$ & 1.6 & 1.7 & 1.7 & 1.7 \\
$\chi^{2} /$ d.o.f. & $619 / 260$ & $302 / 260$ & $265 / 260$ & $273 / 258$ \\
\hline
\end{tabular}

Notes. ${ }^{(a)}$ Implemented with BXA fitting in pyXSPEC. ${ }^{(b)}$ Hydrogen column density $N_{\mathrm{H}}\left[10^{22} \mathrm{~cm}^{-2}\right]$, temperature kT [keV], power-law photon index $\Gamma$, X-ray flux $F_{\mathrm{X}}(0.5-10 \mathrm{keV}) 10^{-12} \mathrm{erg} \mathrm{s}^{-1} \mathrm{~cm}^{-2}$ (uncertainties $\sim 3 \times 10^{-14} \mathrm{erg} \mathrm{s}^{-1} \mathrm{~cm}^{-2}$ ), and goodness of fit $\left(\chi^{2}\right)$ with degrees of freedom (d.o.f.) in the fit. ${ }^{(c)}$ The metal abundance is fixed to solar.

Using the best-fit TBabs $\times$ bremss model the $X M M$ Newton count rate of $\sim 0.3 \mathrm{cts}^{-1}$ translates into a flux of $1.7 \times 10^{-12} \mathrm{erg} \mathrm{s}^{-1} \mathrm{~cm}^{-2}$ (here $0.5-7.0 \mathrm{keV}$, for comparison with Chandra). The $3 \sigma$ upper limit from previous Chandra observations of $\sim 0.005 \mathrm{ct} \mathrm{s}^{-1}$ translates into $8.6 \times 10^{-14} \mathrm{erg} \mathrm{s}^{-1} \mathrm{~cm}^{-2}$ $(0.5-7.0 \mathrm{keV})$. This means that the source flux varies by a factor of $\gtrsim 20$ on long timescales $(\sim 10 \mathrm{yr}$ between upper limit and detection). Within the XMM-Newton EPIC PN observation, the flux varies significantly $(\sim 5 \sigma)$ by up to a factor of $\sim 4$ on timescales of minutes ( $77 \mathrm{~s}$ binning, see Fig. 3 ). The source is also covered as part of the Swift Galactic bulge survey (Shaw et al. 2017) with the Neil Gehrels Swift Observatory 


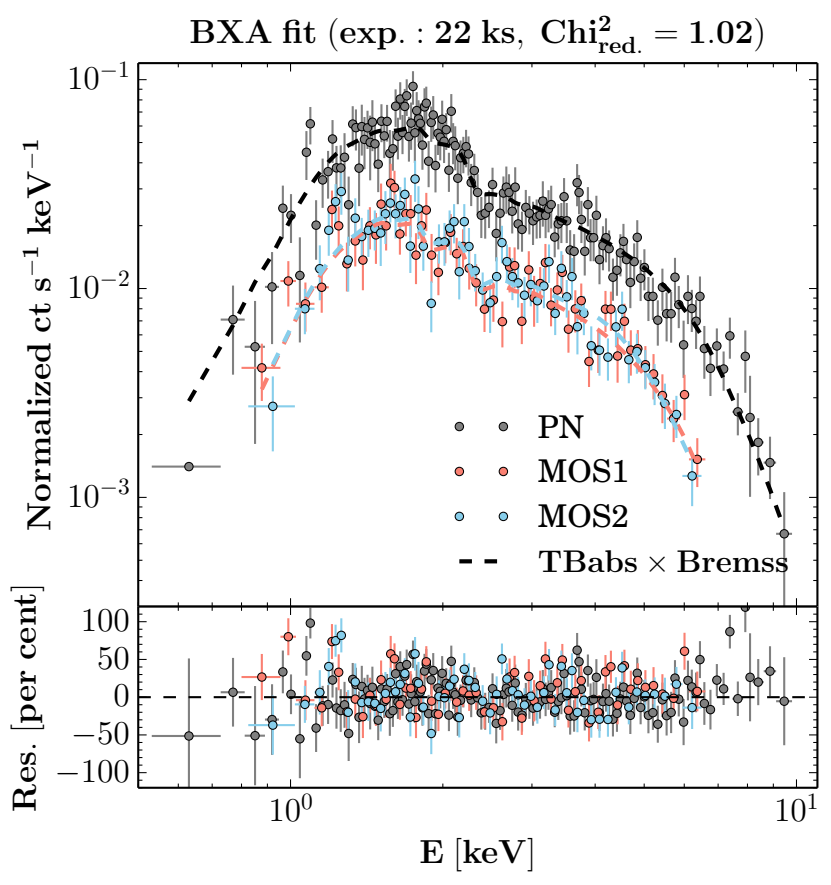

Fig. 2. XMM-Newton EPIC spectrum $(0.5-10 \mathrm{keV})$ for PN, MOS1, and MOS2 separately with residuals of a TBabs $\times$ bremss model fitted using BXA in pyXSPEC.

X-ray telescope (for a mission description, see Burrows et al. 2005) in 2017 and 2018, but the 60-120 s exposures did not allow us to significantly constrain the long-term light curve. Recent Swift ToO observations (ObsIDs: 00010652001/2, 201804-08/10) and a renewed coverage within the XMM-Newton GC/GB survey (ObsID: 0801682101, 2018-03-18) provide an upper limit of $F_{\mathrm{X}} \lesssim 7.2 \times 10^{-14} \mathrm{erg} \mathrm{s}^{-1} \mathrm{~cm}^{-2}$ (a factor of $\gtrsim 20$ fainter again).

\subsection{X-ray periodicity}

We extracted X-ray light curves for source and background from the same regions as the spectra for $24.7 \mathrm{ks}$ of observation with the PN, MOS1, and MOS2 instruments. We used standard event filtering, corrections for barycentric photon arrival time, and the same good time intervals (GTI) to obtain background-subtracted, vignetting-corrected $0.5-10 \mathrm{keV}$ light curves from all instruments.

We performed a Lomb-Scargle analysis (Scargle 1982) with the periodogram tool provided by the NASA exoplanet archive $^{2}$ and found a peak at $\sim 511 \mathrm{~s}$ in the unbinned PN data (confirmed by the MOS1 data at lower significance because of its smaller effective area). We discard MOS2 in the following analysis because the background is higher and less stable $^{3}$. The period was found independent of the light-curve binning.

For a more detailed significance analysis, $77 \mathrm{~s}$ binning was chosen as a compromise between time resolution and measurement uncertainty in each bin. Figure 3 shows the light curve of PN+MOS1 and the power spectral density (PSD) calculated for 161 frequencies (total power: $1213 \mathrm{ct}^{2} \mathrm{~s}^{-1}$ ) with the fast

\footnotetext{
2 https://exoplanetarchive.ipac.caltech.edu/docs/ tools.html

3 MOS2 showed a significantly more noisy background PSD (corrupting the source periodogram) than PN and MOS1.
}

Fourier transform (FFT) periodogram function of the scipy Python package (version 0.17.1). To estimate the significance of the $511 \mathrm{~s}$ peak, we simulated $10^{6}$ light curves with the root mean square (RMS) amplitude normalised to the measured signal. White-noise simulations best reproduced the observed PSD, leading to the flat significance contours shown in Fig. 3. The most significant peak is located at $511 \pm 10 \mathrm{~s}(\sim 3.7 \sigma)$, consistent with the independent Lomb-Scargle analysis (see above). In addition, we performed simulations with a pink-to-red noise PSD, breaking at a period of $500 \mathrm{~s}$ (typical for CVs, e.g., Dobrotka et al. 2014). The results indicate that even in this case, the periodicity is significant at $\sim 3.9 \sigma$.

Figure 4 shows the PN light curve folded by a 511 s period. The resulting phase diagram is best fit by a sine function with amplitude $0.17 \pm 0.02$ (translating into $\sim 17 \%$ pulsed fraction). The fit to a constant value results in $\chi^{2} /$ d.o.f. $\approx 39.2 / 6$, which is further evidence for variability with the folded period.

\subsection{Correlation with optical sources}

The simultaneous XMM-Newton optical monitor observation could not be used for counterpart constraints because the source was outside its field of view. The closest correlation at $\sim 1.5^{\prime \prime}$ ( $\sim 1 \sigma$ uncertainty) of the X-ray position is 2MASS 175035102935562 (Cutri et al. 2003) with $\sim 13(J, H, K)_{\text {mag }}$ (Fig. 1, bottom left). The Yale/San Juan Southern Proper Motion Catalogue (Girard et al. 2011) provides a proper motion of $\sim 80 \mathrm{mas} \mathrm{yr}^{-1}$ for the 2MASS source, which would make it most likely a foreground M star. The VISTA Variable in the Via Lactea Survey DR2 (Minniti et al. 2017) provides $\sim 16,15,14,13(Z, Y, J, H)_{\mathrm{mag}}$ for the position.

The 2MASS position is resolved into two optical sources in the Chandra Galactic bulge survey (Wevers et al. 2016), and the OGLEII survey (Udalski et al. 2002) - both 21, $17(V, I)_{\mathrm{mag}}$ (sources 1 and 2, Fig. 1, bottom). The OGLEII light curves (about one observation per day) of possible counterparts show no significant variability, but this does not exclude intrinsic variability because the uncertainties are large.

The Gaia DR2 catalogue (Gaia Collaboration 2018) constrains the distance to source 1 (Fig. 1, bottom) to $\sim 0.8-2.2 \mathrm{kpc}$ and to source 2 to $\gtrsim 1.4 \mathrm{kpc}$, with proper motions of 14 and 11 mas yr $^{-1}$ respectively, and both have $G_{\mathrm{mag}} \sim 19$.

\section{Discussion}

\subsection{IP properties from $X$-rays}

In the XMM-Newton GC/GB scan of currently $\sim 6 \mathrm{deg}^{2}$, XMMU J175035.2-293557 is a rare X-ray source with a hard spectrum, strong variability, and intermediate brightness.

A bremsstrahlung model is the best fit to the X-ray spectrum, which together with the evidence for a $511 \mathrm{~s}$ period is a strong indication that the source is an IP. The best-fit temperature of a brems model is $13.9 \pm 2.5 \mathrm{keV}$, which would suggest a WD mass of about $0.4-0.5 M_{\odot}$ (this is at the low end of the known mass function, see Ritter \& Kolb 2003; Brunschweiger et al. 2009) with a radius of about $0.01 R_{\odot}$ and a shock height of the accretion column of $\sim(1-2) \times 10^{6} \mathrm{~cm}$ (Yuasa et al. 2010). The hard spectrum of the source would fit in the class of IPs with hard X-ray emission (Haberl \& Motch 1995), but the high absorption at lower energies could obscure an additional softer emission. The significance of the $500 \mathrm{~s}$ periodicity is above $3 \sigma$ in the soft $0.5-2.0 \mathrm{keV}$, but only $\sim 2 \sigma$ in the hard $2.0-10 \mathrm{keV}$ band. This would be expected in IPs, which usually show a higher variation 

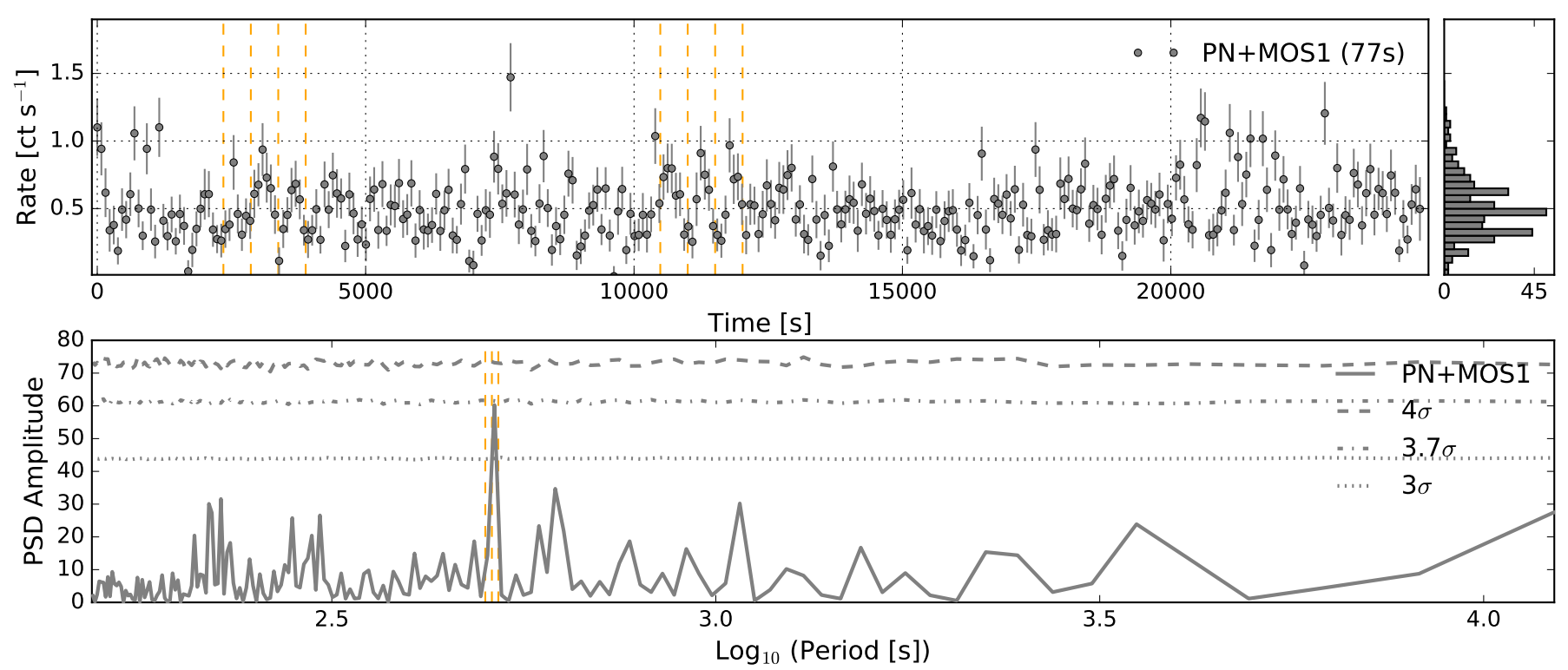

Fig. 3. Top: background-subtracted, vignetting-corrected X-ray ( $0.5-10 \mathrm{keV})$ light curve of XMM-Newton EPIC PN+MOS1, binned to $77 \mathrm{~s}$. Dashed orange lines mark intervals where the $511 \mathrm{~s}$ pulsations are visible by eye, and the histogram shows the distribution of rates. Bottom: PSD amplitude $\left[\right.$ Rate $\left.^{2} \mathrm{~Hz}^{-1}\right]$. Dashed lines show the confidence contours of simulated light curves without periodicity, assuming white noise variability. The orange dashed lines indicate the $511 \pm 10 \mathrm{~s}$ period.

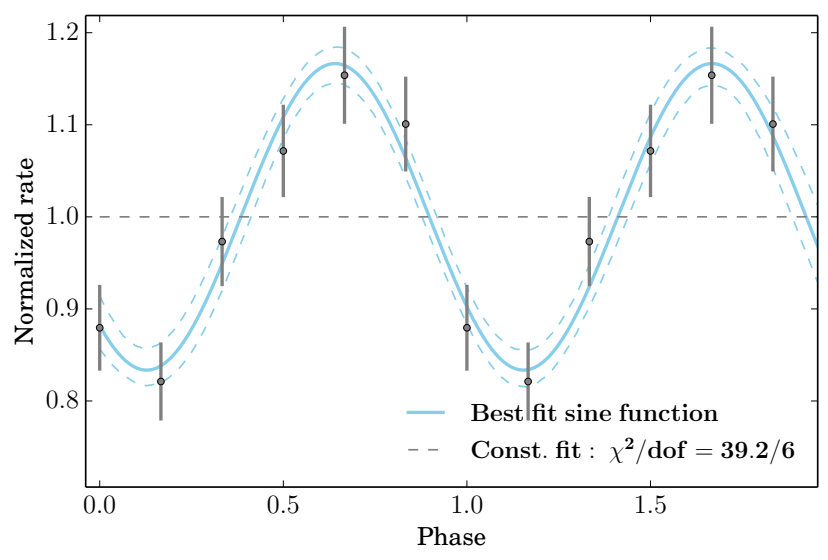

Fig. 4. Normalised rate over phase for the XMM-Newton EPIC PN light curve (77 s bins) folded by a $511 \mathrm{~s}$ period. The best-fit sine function is displayed in blue (uncertainty from Monte Carlo simulations shown in dashed blue). The annotations give the goodness of fit to a constant value of 1.0 (dashed grey line).

amplitude in the softer X-ray band (e.g., Luna et al. 2018). The residuals of the bremsstrahlung model (see Fig. 2) do not show evidence for an Fe-line complex between 6 and $7 \mathrm{keV}$, which is often observed for IPs (e.g., Muno et al. 2004; Revnivtsev et al. 2004). The upper limits on the EW of the main Fe emission lines are consistent with previously discussed IP candidates (e.g., Hong et al. 2009). The X-ray luminosity is in the expected range, but it places the source among the most distant known IPs with some candidates in the GB (see, e.g., Hong et al. 2009; Britt et al. 2013; Torres et al. 2014; Johnson et al. 2017) or sources identified from periodicity in extragalactic novae (in M31, see, e.g., Pietsch et al. 2011). The expected orbital period in the IP scenario would be $P_{\text {orb }} \gtrsim 5000 \mathrm{~s}\left(P_{\text {spin }} / P_{\text {orb }} \lesssim 0.1\right.$, Norton et al. 2004). The light curve shows a hint of modulation on timescales of $\sim 5000 \mathrm{~s}$, but because the observation time was only $25 \mathrm{ks}$, no significant detection of an orbital period was found.

\subsection{Source location and luminosity}

The measured X-ray absorption is consistent with the expected total column density of the Galaxy $\left(\mathrm{N}_{\mathrm{H}}=\mathrm{N}_{\mathrm{HI}}+2 \mathrm{~N}_{\mathrm{H}_{2}}\right.$ $\approx 9 \times 10^{21} \mathrm{~cm}^{-2}$, Willingale et al. 2013) toward the source position. This provides some evidence that the source is located close to the GC (see, e.g., dust layer distribution study by Jin et al. 2017,2018 ). If the source were closer (in front of the main absorbers toward the GC), it would need to have a high intrinsic absorption.

The expected X-ray luminosity range of IPs is about $3 \times 10^{29}-5 \times 10^{33} \mathrm{erg} \mathrm{s}^{-1}$ (Ruiter et al. 2006), which constrains the distance to the source from its XMM-Newton flux and the Chandra upper limit to about $0.1-8 \mathrm{kpc}$. The average luminosity of the source during the XMM-Newton EPIC observation is $(1.3 \pm 0.1) \times 10^{34} \mathrm{erg} \mathrm{s}^{-1}(0.5-7.0 \mathrm{keV})$ assuming a TBabs $\times$ bremss model (see Fig. 2) and a distance of $8 \mathrm{kpc}$ (distance to the GC, see, e.g., Eisenhauer et al. 2003). The $3 \sigma$ upper limit from previous Chandra observations in this case would be $6.6 \times 10^{32} \mathrm{erg} \mathrm{s}^{-1}(0.5-7.0 \mathrm{keV})$.

For a local IP, a relatively blue optical counterpart would be expected (Hong et al. 2009), but no candidate fitting this criterion was found in the $3 \sigma$ error circle around the source position (see above). Assuming that the true counterpart is below the sensitivity limit of current catalogues $\left(V_{\text {mag }} \sim 22\right)$, with the typical CV magnitude range $M_{\mathrm{V}} \approx 5.5-10.5$ (e.g., Hong et al. 2009), and rising absorption from $A_{\mathrm{V}} \approx 1-4$ (estimated from Schlafly \& Finkbeiner 2011; Schultheis et al. 2014) between 1 and $8 \mathrm{kpc}$, we can constrain its distance to $\gtrsim 1 \mathrm{kpc}$. Using the hydrogen column density measured in the X-ray spectrum, we estimate $A_{\mathrm{V}} \approx 6$ (relation by Güver \& Özel 2009). If the donor star were a subdwarf like in GK Per, one of the faint optical correlations (e.g., numbers 1, 2, or 3 in Fig. 1) might be the true counterpart, which would be consistent with a location in the GB. Hong et al. (2009) found a probable optical counterpart with $21.7 \mathrm{~V}_{\text {mag }}$ for an IP in the GB, but located in Baade's window, where the extinction is only $A_{\mathrm{V}} \approx 1.4$ at $\gtrsim 3 \mathrm{kpc}$. 
Together with the $\mathrm{N}_{\mathrm{H}}$, flux, and optical counterpart constraints, we used the stellar density and Galactic X-ray source distribution (as shown for the GB IP candidate in Hong et al. 2009) to conclude that the new IP candidate is most likely located in the GB $(8 \pm 2 \mathrm{kpc}$, see overview of the GB structure by Bland-Hawthorn \& Gerhard 2016).

\subsection{Alternative interpretations}

Just at the border of the $3 \sigma$ positional error circle is a Be star candidate from the OGLEII survey (Sabogal et al. 2008) for which no significant variability was detected. A Be X-ray binary could show periodic pulsations similar to IPs, but the X-ray spectrum would be well fit by a power law with photon index $\approx 1$ (see, e.g., the population of the Small Magellanic Cloud, Haberl \& Pietsch 2004; Coe \& Kirk 2015; Haberl \& Sturm 2016).

Based on its X-ray luminosity, and flux variation of at least a factor of ten, the newly discovered source is a very faint X-ray transient (VFXT with $L_{\mathrm{X}} \approx 10^{34-36} \mathrm{erg} \mathrm{s}^{-1}$, see, e.g., King \& Wijnands 2006). The luminosity peaks of the source are about $10^{34} \mathrm{erg} \mathrm{s}^{-1}$ if it is located at a GC distance. The variability within the observation does not indicate an ongoing brightening. VFXTs are a still poorly explored family of transients that are expected to be mostly X-ray binaries (with a neutron star or black hole as compact object) with a low accretion rate from a low-mass donor star at $\lesssim 10^{-13} M_{\odot} \mathrm{yr}^{-1}$ (King \& Wijnands 2006; Maccarone \& Patruno 2013; Heinke et al. 2015).

We note that the spectrum of XMMU J175035.2-293557 is harder than for typical VFXTs (see, e.g., Armas Padilla et al. 2013), and periodic pulsations are usually not observed in VFXTs. Our results show that highly variable IPs might contribute to the faint end of the VFXT population.

\section{Conclusions}

With the results of this work, we constrained the nature of the newly discovered X-ray source XMMU J175035.2-293557. The most likely scenario is that it is an intermediate polar in the Galactic bulge. It is one of the most luminous candidates, which implies a strong magnetic field of the white dwarf. The spin period of the white dwarf would be $P_{\text {spin }}=511 \pm 10 \mathrm{~s}$, but the orbital period of the system $P_{\text {orb }}$ could not be recovered from the current data. The system was a factor of $\gtrsim 20$ fainter in observations $\sim 10$ yr before and $\sim 6$ months after the first detection. This transient nature makes it most likely the second member of the GK Per class. The X-ray spectra are best fit by a bremsstrahlung model with a temperature of $13.9 \pm 2.5 \mathrm{keV}$. No optical counterpart is identified, but several candidates are found. The true counterpart might be below the detection threshold of currently available surveys. With an average flux of $\sim 1.7 \times 10^{-12} \mathrm{erg} \mathrm{s}^{-1} \mathrm{~cm}^{-2}$, this type of source will also be detected in the upcoming eROSITA all-sky survey (Merloni et al. 2012), which will allow studying a larger fraction of the Galactic population (on the order of 100 source are expected). Followedup with high-resolution X-ray observatories that have large effective areas, such as ATHENA (Nandra et al. 2013), the spin and orbital periods of similar IP systems will be significantly detected.

Acknowledgements. We thank the anonymous referee for very constructive comments that helped to improve the clarity of the paper. We thank M. Freyberg and C. Wegg for helpful discussions. These results are based on observations obtained with XMM-Newton, an ESA science mission with instruments and contributions directly funded by ESA Member States and NASA. We acknowledge the use of public data from the Swift data archive and thank the Swift team for scheduling the ToO observation; data obtained from the Chandra Data Archive and the Chandra Source Catalog, and software provided by the Chandra Xray Center (CXC) in the application package CIAO; NASA's Astrophysics Data System; the VizieR catalogue access tool, CDS, Strasbourg, France; SAOImage DS9, developed by Smithsonian Astrophysical Observatory; data and/or software provided by the High Energy Astrophysics Science Archive Research Center (HEASARC), which is a service of the Astrophysics Science Division at NASA/GSFC and the High Energy Astrophysics Division of the Smithsonian Astrophysical Observatory; the SIMBAD database, operated at CDS, Strasbourg, France; NASA's SkyView facility located at NASA Goddard Space Flight Center; the NASA Exoplanet Archive, which is operated by the California Institute of Technology, under contract with the National Aeronautics and Space Administration under the Exoplanet Exploration Program; the NASA/IPAC Infrared Science Archive, which is operated by the Jet Propulsion Laboratory, California Institute of Technology, under contract with the National Aeronautics and Space Administration; data provided by the Science Data Archive at NOAO. NOAO is operated by the Association of Universities for Research in Astronomy (AURA), Inc. under a cooperative agreement with the National Science Foundation; use of the python packages Matplotlib, scipy, numpy, and pyXSPEC. F. Hofmann and G. Ponti acknowledge financial support from the BMWi/DLR grants FKZ 50 OR 1715 and 50 OR 1604.

\section{References}

Armas Padilla, M., Degenaar, N., \& Wijnands, R. 2013, MNRAS, 434, 1586 Arnaud, K. A. 1996, in Astronomical Data Analysis Software and Systems V, eds. G. H. Jacoby, \& J. Barnes, ASP Conf. Ser., 101, 17

Barbera, E., Orlando, S., \& Peres, G. 2017, A\&A, 600, A105

Bland-Hawthorn, J., \& Gerhard, O. 2016, ARA\&A, 54, 529

Britt, C. T., Torres, M. A. P., Hynes, R. I., et al. 2013, ApJ, 769, 120

Brunschweiger, J., Greiner, J., Ajello, M., \& Osborne, J. 2009, A\&A, 496, 121

Buchner, J., Georgakakis, A., Nandra, K., et al. 2014, A\&A, 564, A125

Burrows, D. N., Hill, J. E., Nousek, J. A., et al. 2005, Space Sci. Rev., 120, 165

Calamida, A., Sahu, K. C., Anderson, J., et al. 2014, ApJ, 790, 164

Calamida, A., Sahu, K. C., Casertano, S., et al. 2015, ApJ, 810, 8

Coe, M. J., \& Kirk, J. 2015, MNRAS, 452, 969

Cropper, M. 1990, Space Sci. Rev., 54, 195

Cutri, R. M., Skrutskie, M. F., van Dyk, S., et al. 2003, VizieR Online Data Catalog: II/246

Dobrotka, A., Mineshige, S., \& Ness, J. U. 2014, MNRAS, 438, 1714

Downes, R. A., Webbink, R. F., Shara, M. M., et al. 2001, PASP, 113, 764

Eisenhauer, F., Schödel, R., Genzel, R., et al. 2003, ApJ, 597, L121

Fruscione, A., McDowell, J. C., Allen, G. E., et al. 2006, Proc. SPIE, Conf. Ser., 6270,1

Gaia Collaboration (Brown, A. G. A., et al.) 2018, A\&A, 616, A1

Garmire, G. P., Bautz, M. W., Ford, P. G., Nousek, J. A., \& Ricker, Jr., G. R. 2003, in X-Ray and Gamma-Ray Telescopes and Instruments for Astronomy, eds. J. E. Truemper, \& H. D. Tananbaum, Proc. SPIE, 4851, 28

Girard, T. M., van Altena, W. F., Zacharias, N., et al. 2011, AJ, 142, 15 Graessle, D. E., Evans, I. N., Glotfelty, K., et al. 2007, Chandra News, 14, 33

Grindlay, J., Zhao, P., Hong, J. S., et al. 2003, Astron. Nachr., 324, 57

Güver, T., \& Özel, F. 2009, MNRAS, 400, 2050

Haberl, F., \& Motch, C. 1995, A\&A, 297, L37

Haberl, F., \& Pietsch, W. 2004, A\&A, 414, 667

Haberl, F., \& Sturm, R. 2016, A\&A, 586, A81

Hailey, C. J., Mori, K., Perez, K., et al. 2016, ApJ, 826, 160

Heinke, C. O., Bahramian, A., Degenaar, N., \& Wijnands, R. 2015, MNRAS, 447, 3034

Hellier, C. 2007, in Star-Disk Interaction in Young Stars, eds. J. Bouvier, \& I. Appenzeller, IAU Symp., 243, 325

Hellier, C., \& Mukai, K. 2004, MNRAS, 352, 1037

Hong, J. 2012, MNRAS, 427, 1633

Hong, J. S., van den Berg, M., Laycock, S., Grindlay, J. E., \& Zhao, P. 2009, ApJ, 699, 1053

Jin, C., Ponti, G., Haberl, F., \& Smith, R. 2017, MNRAS, 468, 2532

Jin, C., Ponti, G., Haberl, F., Smith, R., \& Valencic, L. 2018, MNRAS, 477, 3480

Johnson, C. B., Torres, M. A. P., Hynes, R. I., et al. 2017, MNRAS, 466, 129

Jonker, P. G., Bassa, C. G., Nelemans, G., et al. 2011, ApJS, 194, 18

Jonker, P. G., Torres, M. A. P., Hynes, R. I., et al. 2014, ApJS, 210, 18

Kim, Y., \& Beuermann, K. 1995, A\&A, 298, 165

King, A. R., \& Wijnands, R. 2006, MNRAS, 366, L31

Krivonos, R., Revnivtsev, M., Churazov, E., et al. 2007, A\&A, 463, 957

Laycock, S., Grindlay, J., van den Berg, M., et al. 2005, ApJ, 634, L53

Luna, G. J. M., Mukai, K., Orio, M., \& Zemko, P. 2018, ApJ, 852, L8

Maccarone, T. J., \& Patruno, A. 2013, MNRAS, 428, 1335 
Merloni, A., Predehl, P., Becker, W., et al. 2012, ArXiv e-prints [arXiv: 1209.3114]

Minniti, D., Lucas, P., \& VVV Team 2017, VizieR Online Data Catalog: II/348 Mukai, K. 2017, PASP, 129, 062001

Muno, M. P., Arabadjis, J. S., Baganoff, F. K., et al. 2004, ApJ, 613, 1179

Nakashima, S., Nobukawa, M., Uchida, H., et al. 2013, ApJ, 773, 20

Nandra, K., Barret, D., Barcons, X., et al. 2013, ArXiv e-prints [arXiv: 1306.2307]

Norton, A. J., Wynn, G. A., \& Somerscales, R. V. 2004, ApJ, 614, 349

Osborne, J. P. 1988, Mem. Soc. Astron. It., 59, 117

Patterson, J. 1994, PASP, 106, 209

Perez, K., Hailey, C. J., Bauer, F. E., et al. 2015, Nature, 520, 646

Pietsch, W., Henze, M., Haberl, F., et al. 2011, A\&A, 531, A22

Ponti, G., Morris, M. R., Terrier, R., et al. 2015, MNRAS, 453, 172

Pretorius, M. L., \& Mukai, K. 2014, MNRAS, 442, 2580

Revnivtsev, M., Lutovinov, A., Suleimanov, V., Sunyaev, R., \& Zheleznyakov, V. 2004, A\&A, 426, 253

Revnivtsev, M., Sazonov, S., Churazov, E., et al. 2009, Nature, 458, 1142

Ritter, H., \& Kolb, U. 2003, A\&A, 404, 301
Ruiter, A. J., Belczynski, K., \& Harrison, T. E. 2006, ApJ, 640, L167

Sabogal, B. E., Mennickent, R. E., Pietrzyński, G., et al. 2008, A\&A, 478, 659

Scargle, J. D. 1982, ApJ, 263, 835

Schlafly, E. F., \& Finkbeiner, D. P. 2011, ApJ, 737, 103

Schultheis, M., Zasowski, G., Allende Prieto, C., et al. 2014, AJ, 148, 24

Shaw, A. W., Heinke, C. O., Bahramian, A., et al. 2017, AAS/High Energy Astrophys. Div., 16, 400.01

Strüder, L., Briel, U., Dennerl, K., et al. 2001, A\&A, 365, L18

Torres, M. A. P., Jonker, P. G., Britt, C. T., et al. 2014, MNRAS, 440, 365

Turner, M. J. L., Abbey, A., Arnaud, M., et al. 2001, A\&A, 365, L27

Udalski, A., Szymanski, M., Kubiak, M., et al. 2002, Acta Astron., 52, 217

Watson, M. G., King, A. R., \& Osborne, J. 1985, MNRAS, 212, 917

Wevers, T., Hodgkin, S. T., Jonker, P. G., et al. 2016, MNRAS, 458, 4530

Willingale, R., Starling, R. L. C., Beardmore, A. P., Tanvir, N. R., \& O'Brien, P. T. 2013, MNRAS, 431, 394

Wilms, J., Allen, A., \& McCray, R. 2000, ApJ, 542, 914

Yuasa, T., Nakazawa, K., Makishima, K., et al. 2010, A\&A, 520, A25

Yuasa, T., Hayashi, T., \& Ishida, M. 2016, MNRAS, 459, 779 